\title{
Predictive signatures of 19 antibiotics-induced Escherichia coli proteomes
}

Yanbao Yu ${ }^{1}$, Aubrie O'Rourke ${ }^{2}$, Yi-Han Lin ${ }^{1}$, Harinder Singh ${ }^{1}$, Rodrigo Vargas Eguez ${ }^{1}$, Sinem Beyhan ${ }^{2}$ and Karen E. Nelson ${ }^{1,2,}$

1. J. Craig Venter Institute, 9605 Medical Center Drive, Rockville, MD 20850

2. J. Craig Venter Institute, 4120 Capricorn Lane, La Jolla, CA 92037

\section{Supporting Information.}

Figure S1-S7.

Table S3-S5, Excel tables; attached separately.

Table S1-S2. 


\section{More details of machine learning and modeling.}

We explored multiple machine learning methods in WEKA machine learning package, including Random Forest (RF), LibLinear, LibSVM (Support Vector Machine), IBK ((Instance Based Learner) and SMO (Sequential Minimal Optimization). We observed that SMO achieved the best performance (0.855 PRC, 0.9 Precision and 0.898 Recall) as comparing to the other classifiers (Table S1). Next, we applied a supervised attribute filter in WEKA to select minimum set of proteins to achieve high classification. Using CfsSubsetEval as the evaluator algorithm and the BestFirst as the search algorithm in the Weka package we obtained 47 proteins out of 433 as relevant to classification of samples into different MOA groups. We graduly optimized the SMO classifier by removing individual proteins that did not seem to affect the prediction accuracy, which led to 32 proteins. The SMO model with the selected 32 proteins achieved higher classification performance with 0.881 PRC, 0.914 Precision and 0.914 Recall. Next, we remove NA groups from the 443 protein dataset and developed SMO-based prediction model and achieved performance with 0.956 PRC, 0.967 Precision and 0.96 Recall. The attribute filter was used to select minimum set of proteins for classification using NA removed dataset and we obtained a set of 14 proteins. SMO model was built using these 14 proteins and we achieved a high classification of different MOA groups with 0.975 PRC, 0.982 Precision and 0.98 Recall. 

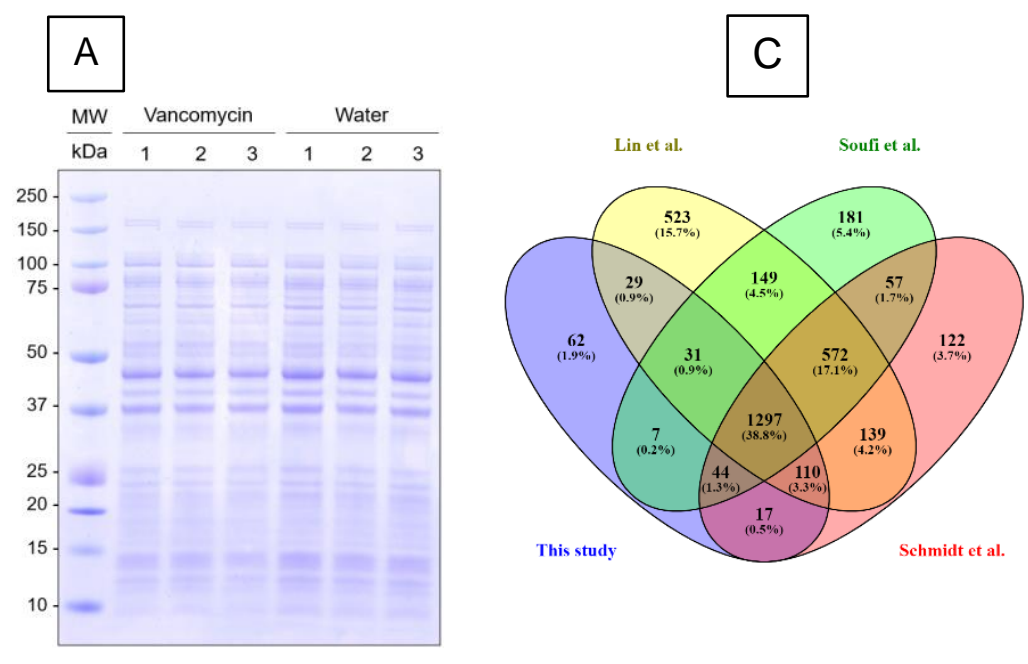

$$
\text { B }
$$

Technical replicates

Digest 1 vs. Digest 2

StageTip 1 vs. StageTip 2
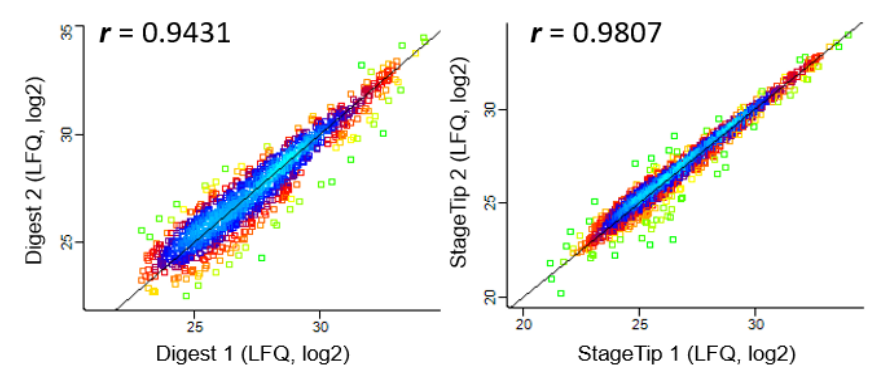

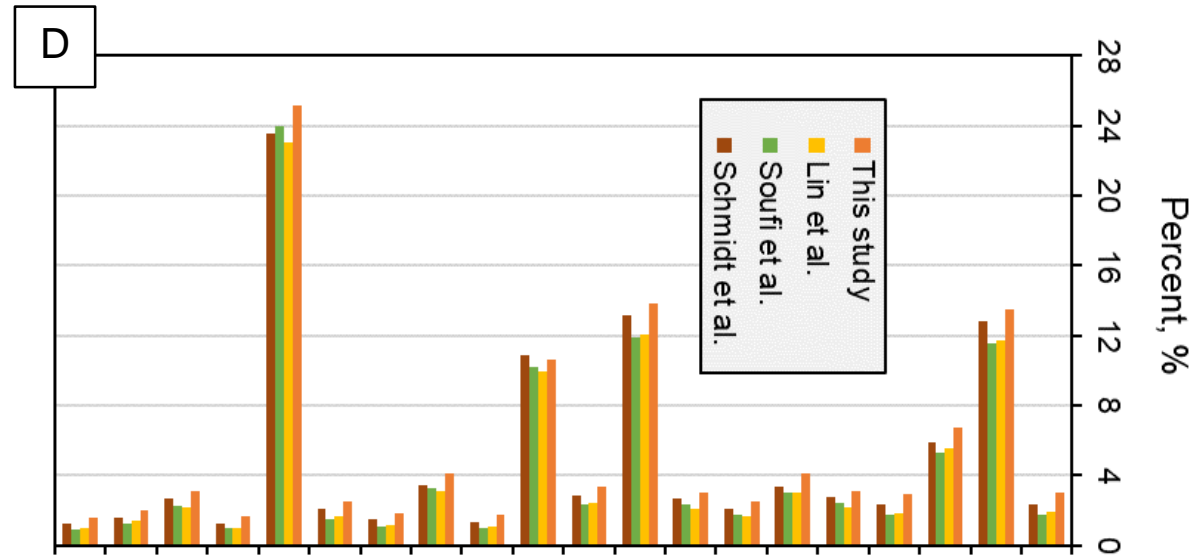

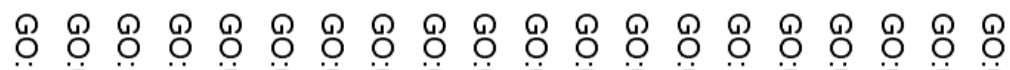

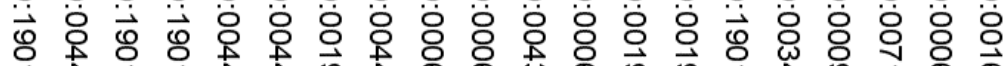

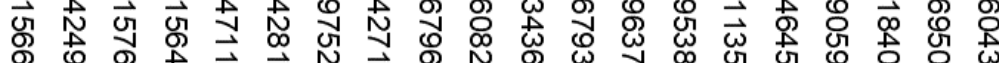

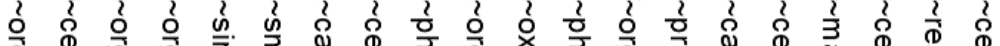

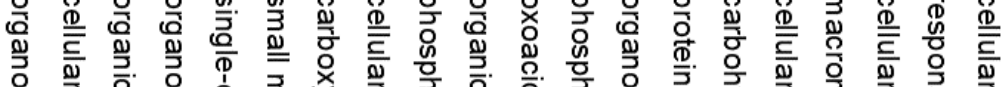

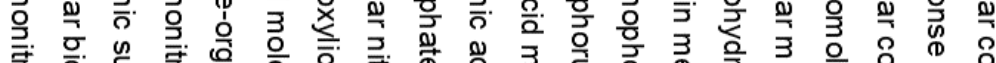

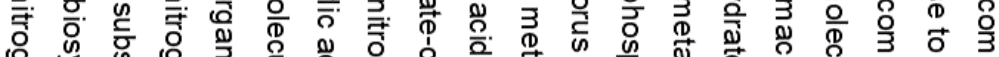

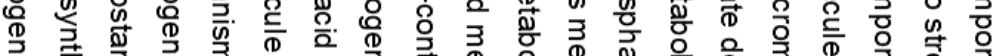

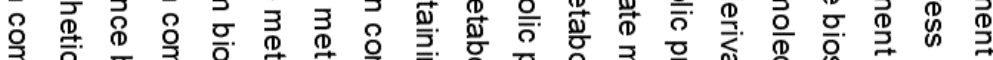

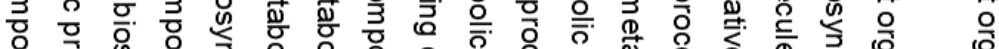

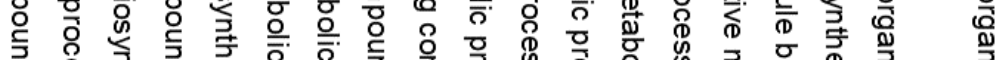

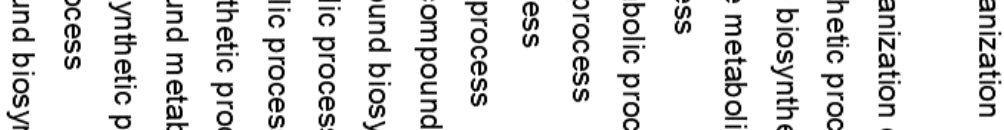

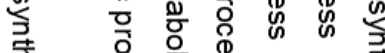
实

产 高品 总 훙 怘
을 勇

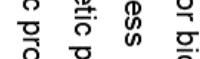

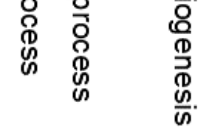


Supplementary Figure S-1. (A) SDS PAGE analysis of protein lysates from vancomycin treated and mock-treated E. coli cells. Three biological replicates were performed for each treatment, and each was processed separately for the gel analysis. High similarities can be seen between different replicates and between different treatments. (B) Gene Ontology analysis of enriched biological process terms between our study and the one by Lin et al. The latter studied the phosphoproteome of E. coli upon antibiotic treatment. The comparison of the two proteomes (around 1,600 vs. 2,500 IDs) were plotted in the context of biological process terms. (C) Density plot of technical replicate of digestion (digest 1 vs. digest 2) and desalting (StageTip1 vs. StageTip2) experiments. The log2 transformed LFQ values were used for the plot. Pearson $r$ values are depicted on the upper left corner of the plots. 


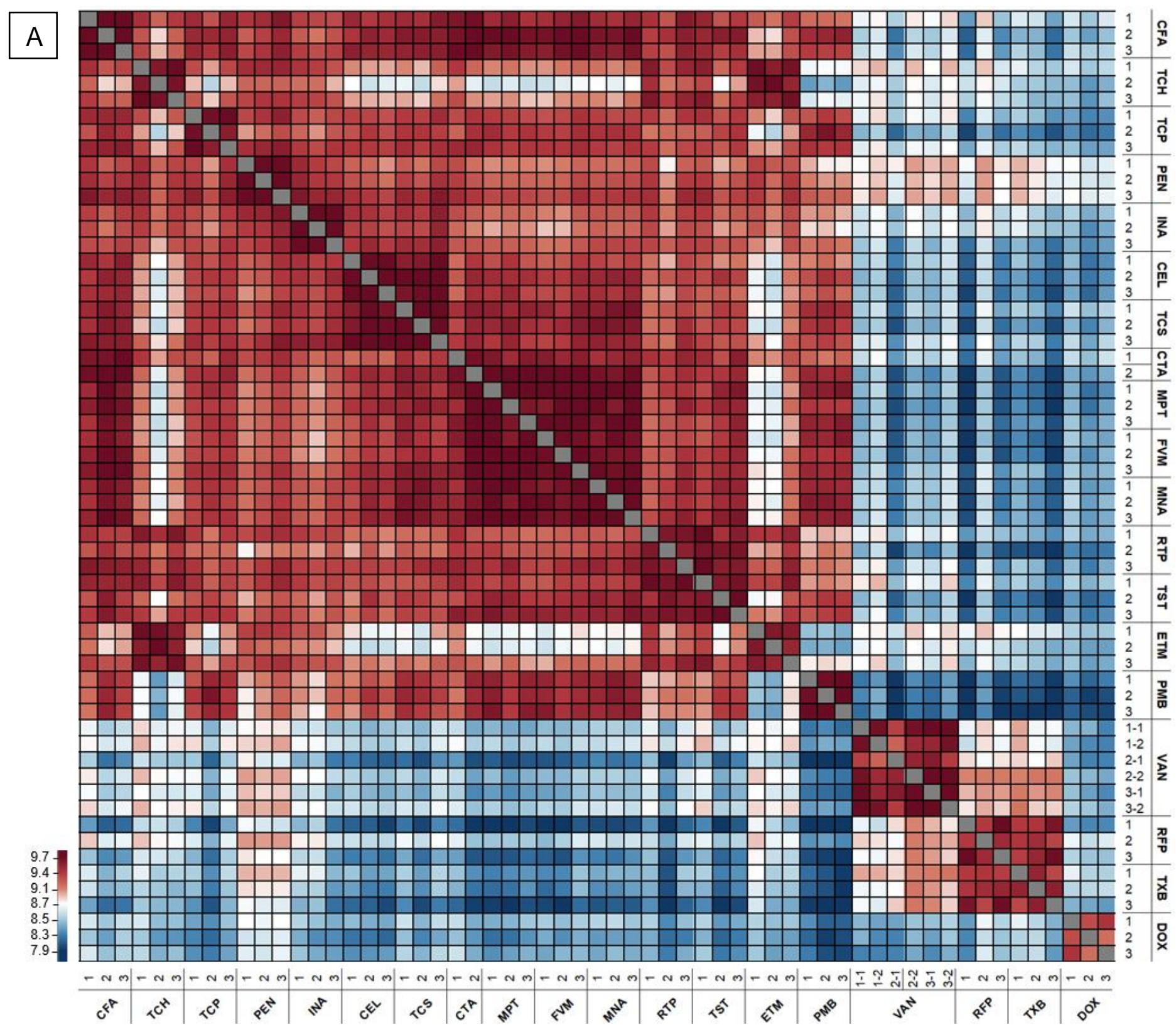



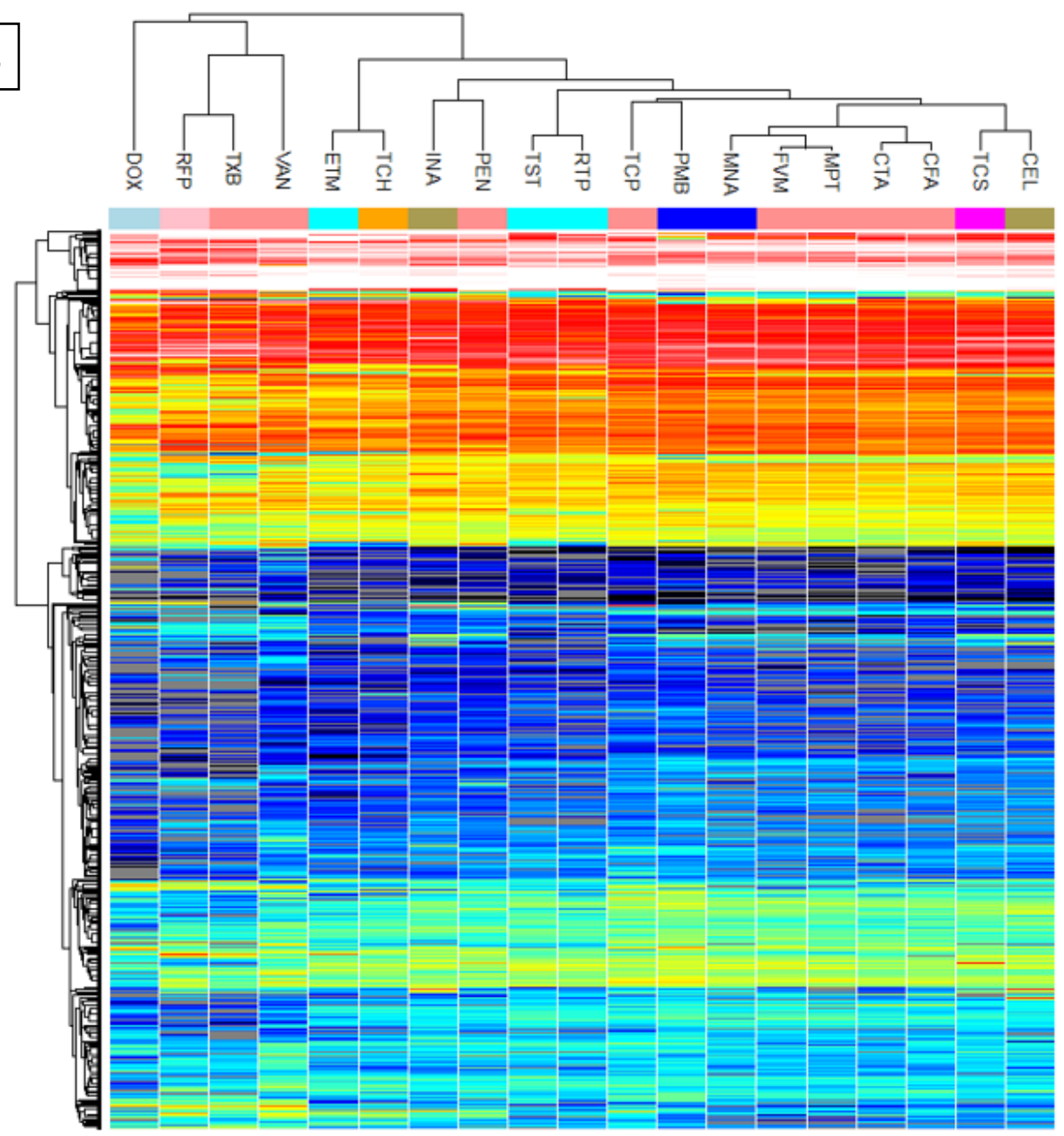

MOA

- Cell wall

- Cell membrane

- Protein synthesis

- Fatty acid synthesis/detergent

- Fatty acid synthesis

- Protein synthesis (30S)

- Protein synthesis (50S)

- RNA synthesis

Relative intensity, $\log 2$

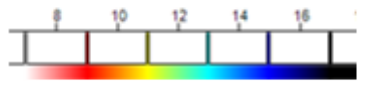


Supplementary Figure S-2. (A) Pearson correlation of E. coli proteomes induced by 19 different antibiotics. Three biological replicates (1, 2 and 3, as shown in the plot) were performed for each antibiotic treatment. The total unfilter proteome $(1,731$ proteins) was used for the correlation analysis. The Pearson $r$ values were color coded in the plot. Complete description of the three-letter abbreviation of antiboitcs can be found in Table. As a note, two successful biological replicates were obtained for ceftriaxone (CTA) treatment. Also, two additional technical replicates were performed for each biological replicate of vancomycin (VAN) treatment. The correlation was generally high between the three biological replicates of the same sample $(\boldsymbol{r}=0.964 \pm 0.016, \mathrm{n}=67)$ or between different samples $(\boldsymbol{r}=0.893 \pm 0.049, \mathrm{n}=1,644)$. (B) Unsupervised hierarchical clustering of $E$. coliproteomes. For the heatmap plot, the data of biological replicates were merged (median), and then were filtered to have at least 800 valid values in each condition, and also to require the proteins to be quantified in at least 10 out of 19 different conditions, which resulted in 1,034 quantifiable proteins. The relative protein intensity was color coded as shown in the scale. Gray colors indicate missing values, which were not imputed for this plot. Abbreviations of antibiotics can be found in Table 1. 


\section{(A) CytoScape-STRING enrichment result:}

\begin{tabular}{|c|c|c|c|c|}
\hline $\begin{array}{l}\text { \# enriched } \\
\text { genes }\end{array}$ & category & description & enriched genes & FDR value \\
\hline 9 & GO Process & alditol metabolic process & dhaK|gatD|gatB|gatY|glpQ|glpA|glpB|glpC|gldA & $5.30 \mathrm{E}-04$ \\
\hline 34 & GO Process & response to stress & $\begin{array}{l}\text { allR|ybgI|dps|cspD|clpA|wrbA|opgG|opgH|dhaM|dhaK|ac } \\
\text { nA|uspE|uspF|ydhQ|btuE|yeaD|dsdX|dsdA|sseA|ung|clpB } \\
\text { |raiA|yggE|fdoI|fdoG|cadB|groS|hsdR|mr|yjiA|osmY|deo } \\
\text { C|deoA|deoB }\end{array}$ & 0.0013 \\
\hline 13 & GO Process & cellular respiration & $\begin{array}{l}\text { cyoB } \mid \text { gltA|cydA|acnA|glpA|glpB|glpC|nuoG||ldD|fdoI|fdo } \\
\text { G|frdC|yjjI }\end{array}$ & 0.0013 \\
\hline 39 & KEGG Pathways & Metabolic pathways & $\begin{array}{l}\text { aceE|gcd||pxA|pepD|cyoB|folD|gltA|cydA|putA|tmk|nagZ| } \\
\text { dhaK|acnA|ydfG|yeaD|manX|eda|gatD|gatB|gatY|cdd|nu } \\
\text { oG|sseA|cysH|gcvT|ansB|garR|IldD|atpG|hemC|yigB|fdoI } \\
\text { |fdoG|gldA|ubiA|aphA|cadA|frdC|deoA }\end{array}$ & 0.0045 \\
\hline 31 & GO Process & oxidation-reduction process & $\begin{array}{l}\text { aceE } \mid \text { gcd } \mid \text { cyoB } \mid \text { folD|ybeX|gltA|cydA|dps|wrbA|putA|acnA } \\
\text { lydfG|btuE|rfbD|gatD|glpA|glpB|glpC|nuoG|ucpA|yfeX|cy } \\
\text { sH|hybC|mdaB|garR|lldD|fdoI|fdoG|gldA|frdC|yjjI }\end{array}$ & 0.0057 \\
\hline
\end{tabular}

\section{(B) DAVID Gene Ontology enrichment result:}

\begin{tabular}{|c|c|c|c|c|c|c|c|}
\hline Category & Count & $\%$ & PValue & Genes & List Total & Pop Hits & Pop Total \\
\hline GOTERM_BP_DIRECT GO:0009061 anaerobic respiration & 9 & 10.58824 & 4.05E-05 & $\begin{array}{l}\text { P0AEL0, POA9C0, POACE0, POA8Q0, } \\
\text { POA996, P32176, P33232, P25516, } \\
\text { P13033 }\end{array}$ & 79 & 56 & 3277 \\
\hline GOTERM_BP_DIRECT GO:0019563 glycerol catabolic process & 4 & 4.705882 & $6.67 \mathrm{E}-04$ & P76015, P0A9C0, P0A996, P13033 & 79 & 8 & 3277 \\
\hline 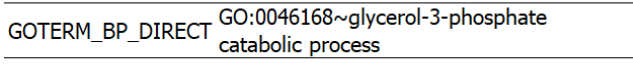 & 3 & 3.529412 & 0.005339 & P0A9C0, P0A996, P13033 & 79 & 5 & 3277 \\
\hline $\begin{array}{l}\text { GOTERM_BP_DIRECT GO:0006974 cellular response to DNA } \\
\text { damage stimulus }\end{array}$ & 12 & 14.11765 & 0.0067 & $\begin{array}{l}\text { P76015, P00926, P0AEL0, P0A6K6, } \\
\text { P24203, P77552, POACN4, P08555, } \\
\text { P37349, P32176, P0A6L0, P07650 }\end{array}$ & 79 & 199 & 3277 \\
\hline $\begin{array}{l}\text { GOTERM_BP_DIRECT GO:0009264 deoxyribonucleotide catabolic } \\
\text { process }\end{array}$ & 2 & 2.352941 & 0.047045 & POA6K6, POA6LO & 79 & 2 & 3277 \\
\hline GOTERM_BP_DIRECT GO:0006986 response to unfolded protein & 2 & 2.352941 & 0.047045 & P63284, P0A6F9 & 79 & 2 & 3277 \\
\hline
\end{tabular}

(C)

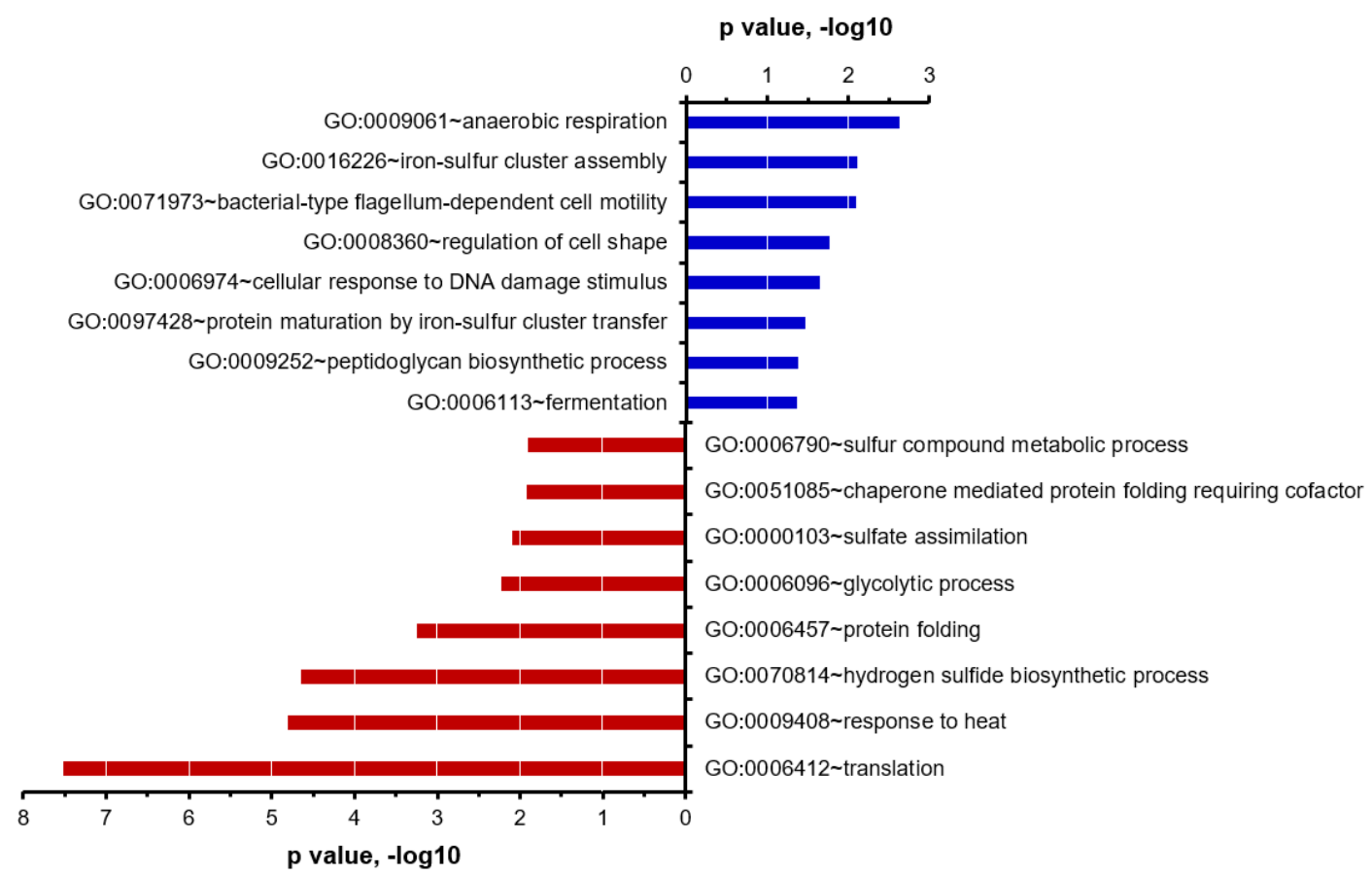


Supplementary Figure S-3. (A) The STRING app in CytoScape (version 3.7.2) and (B) the DAVID Bioinformatics Resources 6.8 (https://david.ncifcrf.gov/) were used for functional enrichment analysis of the 124 differential genes as shown in Figure 2A. (C) Gene Ontology analysis of enriched biological process terms for the two major groups of proteins (up- and down-regulated) shown in Figure 3. 
(A)

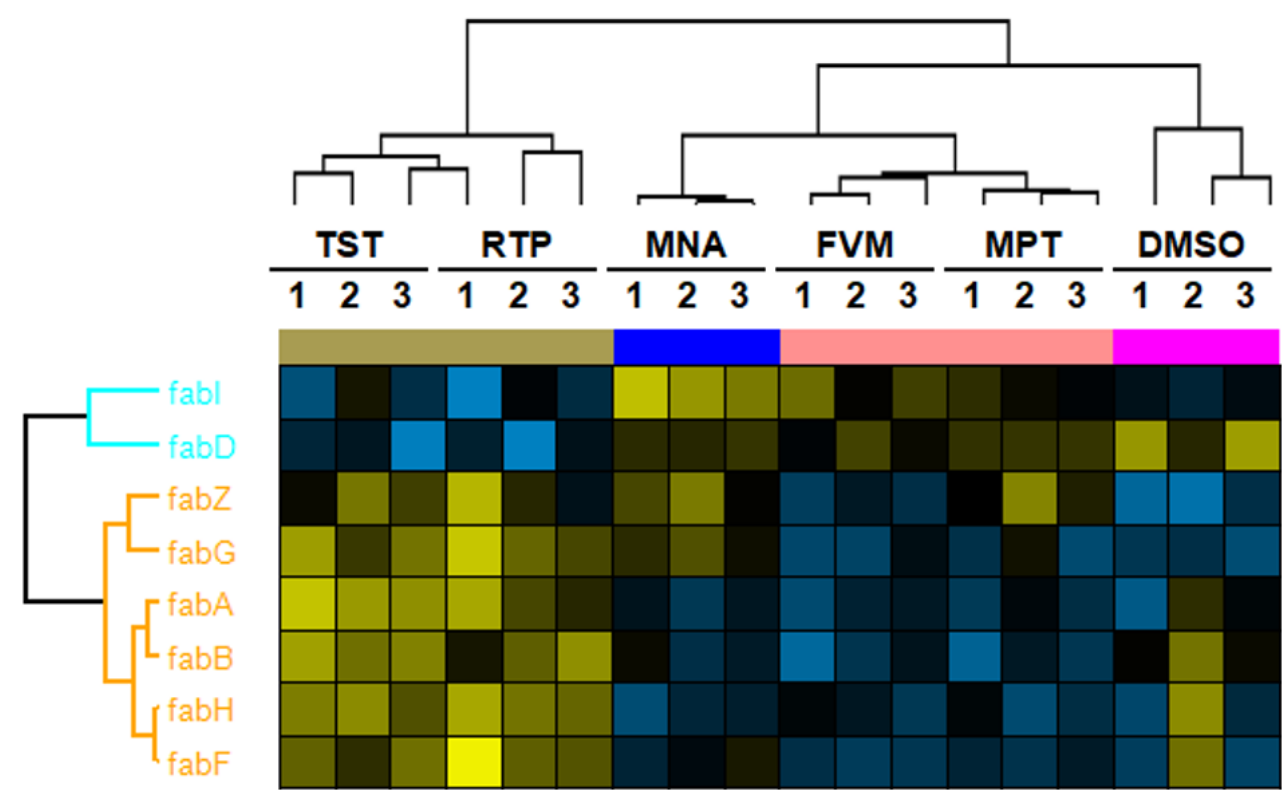

(B)

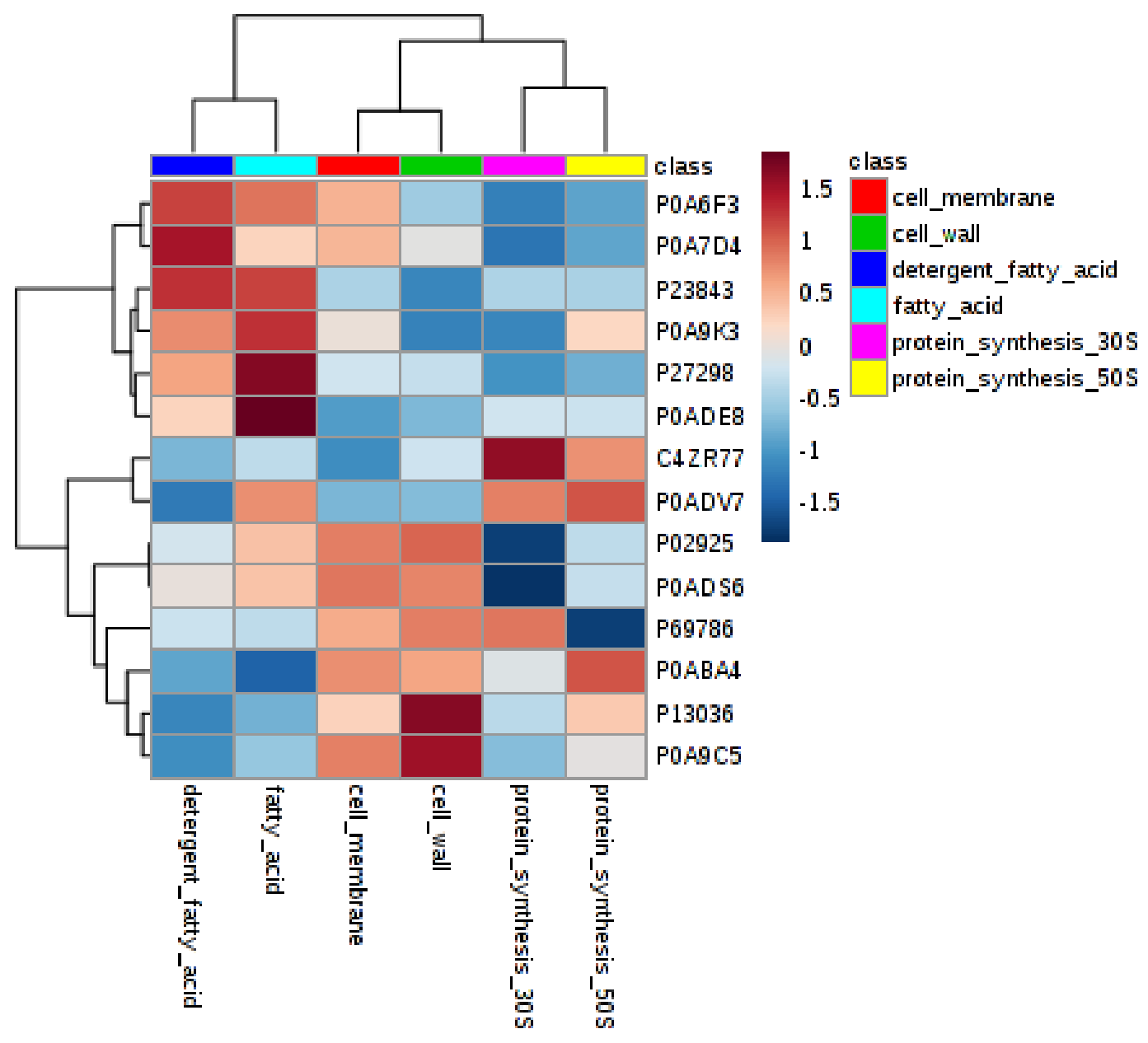


Supplementary Figure S-4. (A) Quantitative profile of 8 fatty acid synthases upon different antibiotics treatment. Details of figure description can be found in Figure 3. Color bar indicates different MOA as described in Table 1. (B). Unsupervised clustering analysis of the 14 proteins and combined MOA classes. 


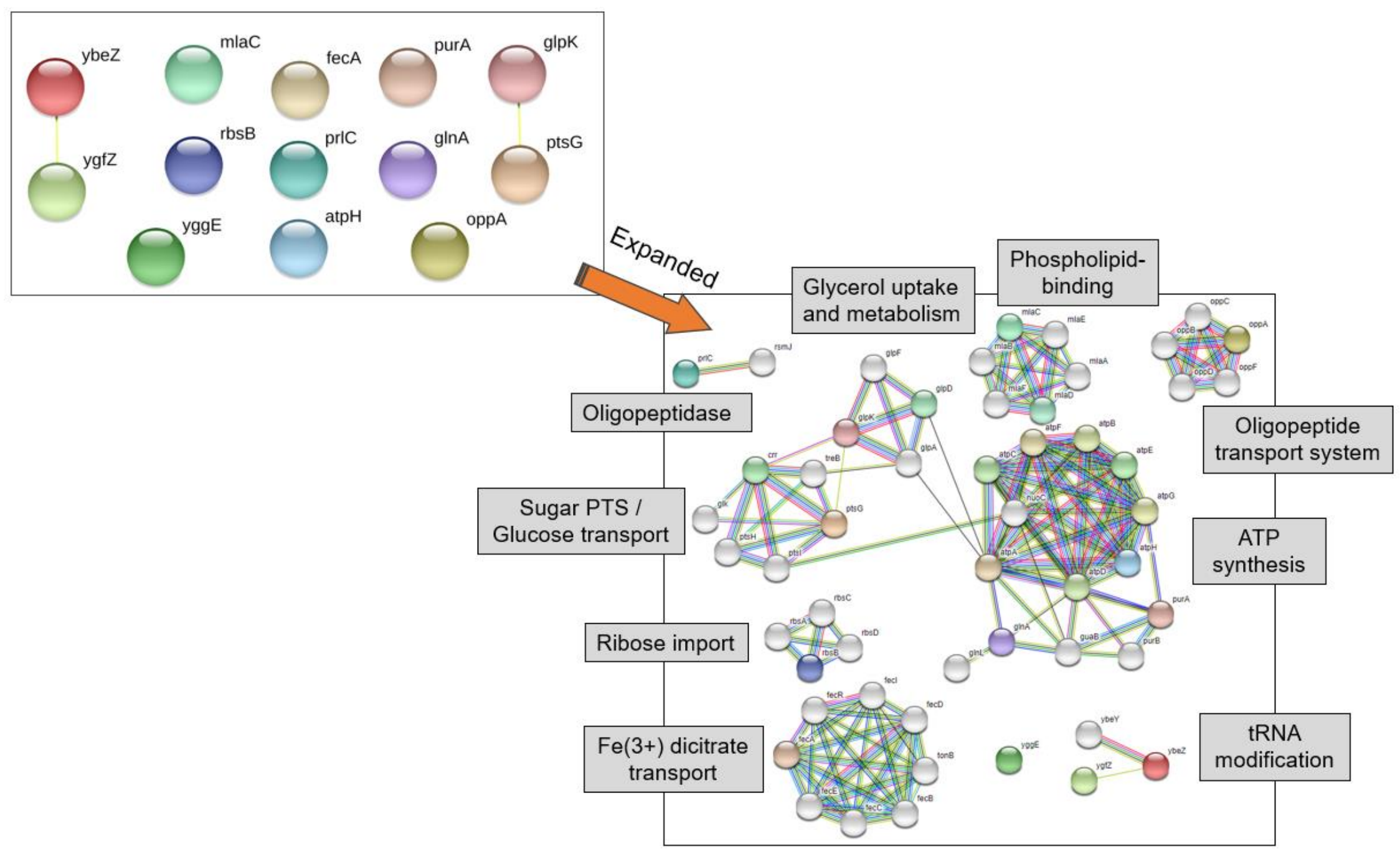


Supplementary Figure S-5. STRING protein network analysis of the panel of the 14 proteins suggested no clear evidence of pathways associated with or interactions between them. Including additional functional proteins, the expanded network showed several isolated protein complexes with few proteins inter-connected between them. 
Supplementary Table S1. Performance classification of different classifiers using 433 proteins. The SMO model performance with 32 proteins and after removing the NA (control treatment by ethanol, water or DMSO) samples from the dataset. The performance of final SMO model included only 14 proteins, achieving overall $98 \%$ accuracy. The model was then tested using the second set of four antibiotics, which achieved overall $87 \%$ accuracy.

\begin{tabular}{|c|c|c|c|c|c|}
\hline & Accuracy (\%) & Precision & Recall & ROC Area & PRC Area \\
\hline$R F$ & 78.57 & NA & 0.786 & 0.956 & 0.835 \\
\hline LibLinear & 87.14 & 0.888 & 0.871 & 0.924 & 0.801 \\
\hline LibSVM & 88.57 & 0.886 & 0.886 & 0.928 & 0.806 \\
\hline IBK & 85.71 & 0.866 & 0.857 & 0.912 & 0.776 \\
\hline \multirow[t]{2}{*}{$S M O$} & 90 & 0.9 & 0.898 & 0.971 & 0.855 \\
\hline & \multicolumn{5}{|c|}{32 proteins } \\
\hline \multirow[t]{2}{*}{ SMO } & 91.42 & 0.914 & 0.914 & 0.971 & 0.881 \\
\hline & \multicolumn{5}{|c|}{ Removed NA } \\
\hline \multirow[t]{2}{*}{$S M O$} & 96 & 0.967 & 0.96 & 0.99 & 0.956 \\
\hline & \multicolumn{5}{|c|}{ Removed NA optimization 14 proteins } \\
\hline \multirow[t]{2}{*}{$S M O$} & 98 & 0.982 & 0.98 & 0.994 & 0.975 \\
\hline & \multicolumn{5}{|c|}{ 14-protein panel applied to set2 } \\
\hline SMO & 86.67 & 0.867 & 0.867 & 0.889 & 0.823 \\
\hline
\end{tabular}


Supplementary Table S2-1. Validation of the predicted model. Confusion matrix of the developed SMO model by the 14protein panel to classify the four antibiotics of set 2 .

\begin{tabular}{ccccc}
\hline MOA class & Cell wall & $\begin{array}{c}\text { RNA } \\
\text { synthesis }\end{array}$ & $\begin{array}{c}\text { Protein } \\
\text { synthesis 30S }\end{array}$ & Accuracy \\
\hline Cell wall & 8 & 1 & 0 & $88.89 \%$ \\
RNA synthesis & 1 & 2 & 0 & $66.67 \%$ \\
Protein synthesis 30S & 0 & 0 & 3 & $100 \%$ \\
\hline
\end{tabular}


Supplementary Table S2-2. Further validation of the 14-protein classifier using the inclusion-exclusion strategy. Basically, we validated our model in these procedures: first, we kept all of the samples of one antibiotic from the same MOA group in the test set, e.g., all of the Monactin samples were kept in the test set 1; similarly, all of Cefotaxime in test set 2 , Meropenem in test set 3, Cerulenin in test set 4 and so on. Then, to compare with the existing model, we used the same set of 14 proteins and the same parameters from the existing SMO model; we did not attempt to optimize the model parameters for the new dataset. The prediction accuracy was high for MOA groups of fatty acid synthesis (100\%), protein synthesis $(100 \%)$ and cell wall $(\geq 85 \%)$, and moderate for cell membrane $(<70 \%)$.

Group 1:
\begin{tabular}{|c|c|c|c|c|c|}
\hline \multicolumn{7}{|c|}{ Polymyxin, Ceftriaxone, Penicillin, Isoniazid, Retapamulin } & \multirow{2}{*}{ Accuracy } \\
\hline & cell_membrane & cell_wall & fatty_acid & protein_synthesis_50S & \\
\hline cell_membrane & 3 & 6 & & & $33.3 \%$ \\
\hline cell_wall & 1 & 19 & & & $95.0 \%$ \\
\hline fatty_acid & & & 6 & & $100 \%$ \\
\hline protein_synthesis_50S & & & & & $100 \%$ \\
\hline
\end{tabular}

Group 2:
\begin{tabular}{|c|c|c|c|c|c|}
\hline \multicolumn{2}{|c|}{ Monactin,Cefotaxime,Meropenem,Cerulenin,Erythromycin } & \multirow{2}{*}{ Accuracy } \\
\hline & cell_membrane & cell_wall & fatty_acid & protein_synthesis_50S & \\
\hline cell_membrane & 5 & 4 & 3 & & $41.7 \%$ \\
\hline cell_wall & 1 & 19 & & & $95.0 \%$ \\
\hline fatty_acid & & & 3 & & $100 \%$ \\
\hline protein_synthesis_50S & & & & 6 & $100 \%$ \\
\hline
\end{tabular}

Group 3:
\begin{tabular}{|c|c|c|c|c|c|}
\hline \multicolumn{7}{|c|}{ Monactin,Flavomycin,Teicoplanin,Cerulenin,Thiostrepton } & \multirow{2}{*}{ Accuracy } \\
\hline & cell_membrane & cell_wall & fatty_acid & protein_synthesis_50S & \\
\hline cell_membrane & 6 & 3 & 1 & 2 & $66.7 \%$ \\
\hline cell_wall & 3 & 17 & & & $85.0 \%$ \\
\hline fatty_acid & & & 5 & & $100 \%$ \\
\hline protein_synthesis_50S & & & & 7 & $100 \%$ \\
\hline
\end{tabular}

